\section{Identification of Causation between Misdiagnosis and Death by the "Loss of Chance" Theory: Two Case Reports}

Keywords: Forensic medicine; Gallbladder carcinoma; Pancreatic carcinoma; Loss of chance; Causation; Degree of contribution

\begin{abstract}
Case 1: A 62-year-old patient was admitted to a provincial hospital for dull epigastric pain and was diagnosed with chronic atrophic cholecystitis, turgid type of gallstone and suspected gallbladder lesion. Two days later a laparotomy and cholecystectomy were performed. The result of the tests on the intraoperative quick diagnosis showed chronic cholecystitis. Several days later the surgeon in charge received the regular paraffin pathological diagnosis report which showed suspected adenocarcinoma of the gallbladder. He did not pay any attention to this report and the patient was not informed of this matter. Consequently the patient visited another hospital and the correct diagnosis, carcinoma of gallbladder, was made. The patient died 8 months later.
\end{abstract}

Case 2: A 54-year-old patient went to a county hospital because of abdominal discomfort and underwent an abdominal computed tomography CT) examination. The results of this examination showed that the body of the pancreas appeared abnormally large in size. The doctor failed to make a correct diagnosis. Later, doctors in another hospital made the diagnoses of suspected carcinoma of the pancreas based on the primary computed tomography imaging mentioned above. The patient eventually died 10 months later. The family members of the deceased held the opinion that the patients deaths resulted from the misdiagnosis, while the hospital held the opposite opinions. Utilizing the theory called "loss of chance" and epidemiological investigations, we, forensic medical experts, poin to the fact that the misdiagnoses were predisposing factors of death, and the degree of contribution should be between $20 \%$ and $40 \%, 10 \%$ and $15 \%$, respectively.

"Loss of chance" is a doctrine permitting recovery of damages for the destruction or reduction of the prospect of achieving a more favorable outcome (of disease). In most medical dispute cases, patients lose their chances of further treatment due to medical fault. If forensic medical experts pay more attention to the seriousness of the disease rather than to the potential influence of the temporal delay in lack of diagnosis, they will be unable to identify the potential influence of a timely correct diagnosis on the outcome (survival). In this paper we present two cases of misdiagnosis. These cases will serve as an introduction of the theory of "loss of chance" as well as an example of its application.

\section{Case Report 1}

\section{Clinical presentation}

On December 19th, 2011 a 63-year-old patient was admitted to a provincial hospital because of epigastric pain that he had been experiencing for about a month. The color Doppler ultrasound imaging and magnetic resonance imaging (MRI) showed turgid type

\section{Journal of}

Forensic

Investigation

\author{
Zhou Shu ${ }^{1,2}$, Chen Jie-Min', Liu Dong-Mei ${ }^{1,3}$, Peng \\ Shu-Ya ${ }^{1,3}$ and Xia Wen-Tao ${ }^{1 *}$

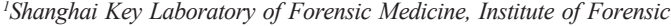 \\ Science, Ministry of Justice, No.1347 West Guangfu Road, Shanghai \\ 200063, China \\ ${ }^{2}$ Department of Forensic Medicine, Shanghai Medical College of Fudan \\ University, Shanghai 200032, China \\ ${ }^{3}$ Department of Forensic Medicine, Medical College of Soochow \\ University, Suzhou 215123, China \\ Address for Correspondence \\ Xia Wen-Tao, Shanghai Key Laboratory of Forensic Medicine, Institute \\ of Forensic Science, Ministry of Justice, No.1347 West Guangfu Road, \\ Shanghai 200063, China, E-mail: xiawentao629@163.com \\ Submission: 22 September, 2014 \\ Accepted: 07 February, 2015 \\ Published: 11 February, 2015
}

of gallstone and atrophic cholecystitis. The turgid type of gallstone had been diagnosed nearly 20 years earlier but no treatment had been given at that time.

The report of the ultrasound scan was recorded as: fatty liver; low level of patchy eco in the right anterior hepatic lobe; turgid type of gallstone in the gallbladder lumen; constrictive gallbladder with the gallbladder wall thickened. The primary diagnoses were chronic atrophic cholecystitis, turgid type of gallstone and suspected not further specified lesion occupying the gallbladder region.

On December 21st, 2011, the serum level of tumor markers was recorded as: all were within the normal range.

On December 21th, 2011, a laparotomy with cholecystectomy was performed, and the diagnosis chronic cholecystitis was made based on an intraoperative frozen section biopsy of the gallbladder wall.

On December 22nd, 2011, the pathological and surgical report of this case stated: the liver appeared normal; the gallbladder and the hepatic portal were surrounded by the greater omentum, which closely adhered to the duodenum; the gallbladder was atrophic and sclerotic, measuring about $5 \times 4 \times 3 \mathrm{~cm}$, the wall measured about $0.5 \mathrm{~cm}$ in thickness; there were 4 mixed gallstones measuring from 0.8 to 1.5 $\mathrm{cm}$ in diameter as well as purulent bile filling the gallbladder lumen; the extrahepatic bile duct measured about $0.9 \mathrm{~cm}$ in diameter. The report of the intraoperative frozen section biopsy of the gallbladder wall stated chronic cholecystitis and no heterocysts were found by the imprint cytologic examination.

On December 25th, 2011, the discharge diagnosis from the provincial hospital was recorded as: chronic cholecystitis and gallstone and the patient were discharged because of improvement in his condition.

On December 26th, 2011, the regular pathological report made 
by the provincial hospital was recorded as: hyperphlogosis of the gallbladder; the lesion was considered to be an adenocarcinoma based on the heterotypic adenoid structure seen infiltrating the submucosa and muscularis, with no nervous tissue invaded. A consultation was suggested. However, the attending surgeon failed to pay attention to this pathological report and at that time no further actions were taken.

On February 27th, 2012, when the patient was admitted to the provincial hospital again, a CT scan showed thickening of the common bile duct wall, dilatation and obstruction of the bile duct, low density in the left lobe of the liver and a lesion in the anterior part of the right hepatic lobe, focal thickening of the fundal stomach wall, and post-surgical signs with the history of cholecystectomy. The doctor in charge, however, neglected to obtain the previous patient record of cholecystectomy and failed to read over the pathological report as well as informing the patient's family of his condition. On that basis, percutaneous transhepaticcholangial drainage (PTCD) was performed with no obvious improvement of the patient's condition.

This time, the diagnosis was obstructive jaundice, a not further specified lesion occupying the regions of distal common bile duct and the liver and signs of post-cholecystectomy with the history of cholecystectomy.

On March 5th, 2012 the patient was transferred to another hospital bringing with him his medical record and the histopathological sections from the previous hospital. The medical history in the primary hospital was recorded as: with reference to reports of the histopathological sections of the lesion, the enhancement computed tomography (CT) scan and positron emission tomographycomputed tomography (PET-CT) scan, the diagnosis was carcinoma of the gallbladder with cholecystectomy performed, invasion of the extrahepatic bile duct, lymphatic metastasis to the hepatoduodenal ligament, obstructive jaundice and post-surgical changes compatible with PTCD.

The patient was treated with gastrointestinal decompression and total parenteral nutrition. Radical surgery was not indicated and a metal stent was inserted in the duodenum.

On August 23th, 2012, the patient passed away. According to the death certificate the cause of death was multiple system organ failure as the result of multiple gallbladder carcinoma metastases.

\section{Forensic medical investigation}

On December 28th, 2012, the forensic medical experts investigated the case. The results were presented as follows:

In the opinion of the deceased family members, when the patient was first admitted to the provincial hospital and received the cholecystectomy, the early stage carcinoma of the gallbladder he suffered from was never identified. The surgeon in charge did not make the correct diagnosis in a timely manner. Consequently, the patient's condition deteriorated. Failure of the surgeon to pay proper attention to the pathological report caused the exacerbation of the condition. It was the misdiagnosis made by the surgeon that caused the death of the patient. The surgeon should assume the compensatory liability.
The hospital insisted that: considering that the condition and pathological presentation of the gallbladder carcinoma was atypical and that the gallbladder carcinoma was at middle-late stage when the patient was hospitalized, it was almost impossible to radically cure the patient and within a short period of time the severe condition would eventually lead to death regardless of treatment. The surgeon did neglect the pathological diagnosis, but that mistake did not directly cause the patient's death.

The images of the abdominal CT scan without contrast made on February 25th, 2012 and the abdominal CT scan with contrast made on February 27th, 2012 showed signs of previously performed cholecystectomy, dilatation of the intrahepatic duct and the common bile duct, low density in the medial segmental duct of the right lobe of the liver, no enhancement signs via contrast enhancement CT scan and no hepatic portal lymphadenopathy or intra-abdominal mass.

The histopathological sections of the lesion showed masses of inflammatory cells on the gallbladder wall, infiltration of a heterotypic adenoid structure in the submucosa and muscularis and carcinoma of the gallbladder.

\section{Conclusion}

In light of the patient's present primary medical record, the diagnosis of the gallbladder carcinoma with invasion beyond the gallbladder muscularis (stages II and III) should have been correctly made. On that basis, the patient should have been treated with radical surgery, including cholecystectomy and a wedge resection of the liver bed plus regional lymphadenectomy [1]. Therefore, cholecystectomy was not adequate and the unperformed radical approach had a certain influence on the patient's prognosis. It is reported that for the patients who undergo radical surgery the overall 5-year survival rates are $34 \%$ and $12 \%$ respectively for stage IA-IB and IIA-IIB. Five-year survival rate for patients without and with lymph node involvement is $37 \%$ and $15 \%$ respectively [2].

The unsuspected malignancy was the primary cause of his death. The surgeon from the provincial hospital failed to inform the patient of the proper diagnosis and as a consequence, the patient suffered a loss of chance of a radical operation. By analyzing the facts of the case and by assessing the epidemiological characteristics of the disease, we, forensic medical experts, came to the conclusion that the misdiagnosis was a contributory cause of death, and the degree of contribution for the provincial hospital should be within a range between $20 \%$ and $40 \%$.

\section{Case Report 2}

\section{Clinical presentation}

On January 25th 2010, a 55 year old man was omitted to the county hospital because of abdominal discomfort and flatulence. The CT imaging record from the county hospital was: no abnormalities or abnormal density shadow of the upper and middle abdomen.

On June 15th 2010, an MRI performed at another hospital which was affiliated to a medical college showed a hypovascular mass in the tail of the pancreas and a hypo dense area in the liver. The findings were found to be consistent with carcinoma of the pancreas with liver metastases. 
Citation: Shu Z, Jie-Min C, Dong-Mei L, Shu-Ya P, Wen-Tao X. Identification of Causation between Misdiagnosis and Death by the "Loss of Chance" Theory: Two Case Reports. J Forensic Investigation. 2015;3(1): 5.

ISSN: 2330-0396

Table 1: Identifications of degree of contribution of medical fault.

\begin{tabular}{|c|c|c|c|}
\hline Type & Range of degree & Average of degree & Meaning \\
\hline $\begin{array}{l}\text { There is no causation between } \\
\text { medical treatment and medical } \\
\text { damage. }\end{array}$ & $0 \% \sim 4 \%$ & $0 \%$ & $\begin{array}{c}\text { There is not any definite causation between medical fault and medical } \\
\text { damage, since the damage was fully caused by the patient's special physical } \\
\text { constitution, characteristics of the illness or the limits of current medical } \\
\text { treatments. }\end{array}$ \\
\hline $\begin{array}{l}\text { There is causality between medical } \\
\text { treatment and medical damage. The } \\
\text { medical treatment comprises a minor } \\
\text { factor. }\end{array}$ & $5 \% \sim 15 \%$ & $10 \%$ & $\begin{array}{l}\text { Damage is mainly caused by the patients' special physical constitution, } \\
\text { characteristics of the illness or the limits of current medical treatments, but the } \\
\text { damage is facilitated or aggravated by the medical fault. However, the medical } \\
\text { damage is unavoidably even without the medical fault. }\end{array}$ \\
\hline $\begin{array}{l}\text { There is causality between medical } \\
\text { treatment and medical damage. } \\
\text { The medical treatment comprises a } \\
\text { secondary factor. }\end{array}$ & $16 \% \sim 44 \%$ & $30 \%$ & $\begin{array}{l}\text { Damage is mainly caused by the patients' special physical constitution, } \\
\text { characteristics of the illness or the limits of current medical treatments, but the } \\
\text { damage is facilitated or aggravated by the medical fault. However, the medical } \\
\text { damage is likely to occur even without the medical fault. }\end{array}$ \\
\hline $\begin{array}{l}\text { There is causality between medical } \\
\text { treatment and medical damage. The } \\
\text { medical treatment comprises the } \\
\text { same factor as the medical damage. }\end{array}$ & $45 \% \sim 55 \%$ & $50 \%$ & $\begin{array}{l}\text { Damage is closely related to the patients' special physical constitution, } \\
\text { characteristics of the illness or the limits of current medical treatment level. } \\
\text { The medical damage will occur both as an effect of medical fault as well } \\
\text { as objective factors, including the patients' special physical constitution, } \\
\text { characteristics of the illness or the limits of medical treatments. If there was no } \\
\text { medical fault, medical damage would be unlikely to occur. }\end{array}$ \\
\hline $\begin{array}{l}\text { There is causality between medical } \\
\text { treatment and medical damage. The } \\
\text { medical treatment comprises the } \\
\text { major factor. }\end{array}$ & $56 \% \sim 95 \%$ & $70 \%$ & $\begin{array}{l}\text { Medical fault was the primary cause or the underlying cause of the damage, } \\
\text { while the patients' special physical constitution, characteristics of the illness or } \\
\text { the limits of current medical treatments are contributory causes. If there was } \\
\text { no medical fault, medical damage would be very unlikely to occur. }\end{array}$ \\
\hline $\begin{array}{l}\text { There is direct causality between } \\
\text { medical treatment and medical } \\
\text { damage. The medical treatment is the } \\
\text { sole contributory factor. }\end{array}$ & $96 \% \sim 100 \%$ & $100 \%$ & $\begin{array}{l}\text { Medical fault was the immediate cause of the damage. If there was no medical } \\
\text { fault, medical damage would definitely not occur. }\end{array}$ \\
\hline
\end{tabular}

On June 18th 2010, the pathological report from the hospital affiliated to a medical college showed a moderately differentiated adenocarcinoma infiltrating the fibrous tissue of the pancreas.

Anti-tumor therapy was performed in the hospital affiliated to a medical college during July $1^{\text {st }}$ to July $26^{\text {th }}$. The patient passed away in November, 2010, ten month after the primary admission to the county hospital.

\section{Forensic medical investigation}

On March 12th, 2013, the forensic medical experts investigated the case. The results were presented as follows:

The deceased family members argued that patient, who suffered from abdominal discomfort and underwent an abdominal CT scan on January 25th, 2010, was told by the county hospital that there were no abnormalities present. However, the diagnosis of the carcinoma of the pancreas was made by another hospital based on the primary CT scan, which was originally made by the county hospital. Subsequently the patient visited more hospital after the diagnosis was made at the second hospital and the diagnosis was the same as that made by the second hospital. However, at that point the patient was incurable and the carcinoma eventually resulted in his death.

The medical organization was of the opinion that the main purpose of the primary CT scan was to find out the cause of abdominal discomfort and flatulence. The diagnosis given by the county hospital was 6 months prior to the one given by the hospital affiliated with the medical college. Computed tomography examination, being nothing more than an adjuvant examination, with its report specifying that the result was for reference only, was not to be considered as the principal mean for the diagnosis of pancreatic carcinoma, which should be established with fine-needle aspiration (FNA) and MRI examination. As the global morbidity and mortality of pancreatic carcinoma was
1:0.99, the medical organization was not liable for the patient's death.

The Abdominal CT scan made on January 25th 2010 showed that the body of the pancreas appeared normal in size. There was a low density mass measuring $2 \mathrm{~cm}$ in maximum diameter and located in the body of pancreas. The peripancreatic fat clearance was normal. The imaging of the arterial, capillary and venous phase was unavailable, because the CT scan was performed without contrast.

\section{Conclusion}

Referring to the abdominal CT report from January 25th 2010, the doctor in charge should have suspected a pancreatic tumor, although he was not able to identify the specific characteristics of the lesion. The doctor should have recommend the patient to have further examinations made, including CT with contrast, MRI examination and serum level of tumor marker [3]. However, the diagnosis "no abdominal abnormalities" was established without adjuvant examinations. Furthermore, the hospital was not up to the adequate standard in medical services, which means the medical service should match the local level of medical services and they didn't attach proper importance to both the obligation to disclose and duty of care. In conclusion, medical fault was obvious. On the other hand, the pancreatic lesion first detected as a low density mass measuring $2 \mathrm{~cm}$ in maximum diameter, was not at an early stage. Based on an analysis of the case facts and on epidemiological knowledge of pancreatic tumors, we assessed that the patient should have been offered surgical excision which might have prolonged his survival period. Pancreatic tumors are highly fatal with an overall 5-year survival rate of less than $4 \%$ [4]. The 5 -year survival rate is $20 \%$ for the selected subset of patients who undergo potential curative surgical resection of the tumor although survival rates as low as $10 \%$ have also been reported [1]. The forensic expert assessed that the misdiagnosis made by the county hospital was partly associated with the patient's death, which 
Citation: Shu Z, Jie-Min C, Dong-Mei L, Shu-Ya P, Wen-Tao X. Identification of Causation between Misdiagnosis and Death by the "Loss of Chance" Theory: Two Case Reports. J Forensic Investigation. 2015;3(1): 5.

occurred relatively rapidly after the initial abdominal discomfort. The degree of contribution for the county hospital should be within the range of $10 \%$ and $15 \%$.

\section{Discussion}

\section{Informed consent and informed option}

According to the Chinese tort law, patient and family members' right to information should receive due respect. This includes explanations by the medical staff about conditions and treatments and the informed consent or choice made by the patients and their family members.

As mentioned in the first case, post-surgical paraffin sections made by the pathological department in the provincial hospital revealed that there was a high probability that the sufferer would develop gallbladder carcinoma and this was formally reported. Due to the hospital's negligence, the patient and his family lost the chance of diagnosis awareness. In accordance, the hospital did not fulfill its lawful obligation to inform. In the second case, although the doctor insisted that the CT scan was for reference only and was not adequate to establish a definite diagnosis, the doctor did not deny that the CT scan had suggested the possibility of pancreatic disease and that further examination should have been recommended. The forensic expert concluded that since obvious changes in the pancreas could be seen on the CT scan, the doctor had neglected these changes and misdiagnosed the patient and hence not fulfilled his responsibility to live up to corresponding standards in medical care. The forensic expert held the opinion that the hospital should have diagnosed the pancreatic disease and informed the patient, but failed to do so. After analyzing both cases we came to the conclusion that the patient should have been offered the chance of further treatment, and that this chance was lost due to the misdiagnosis and the failure to recommend further examination at that time. Eventually, the patients lost their chance of improving their survival rate.

As both cases demonstrate, the patients should have had a chance to be adequately treated based on their initial conditions. Nevertheless, because of the misdiagnoses, both of them lost their opportunities for proper treatment and consequently died.

In summary, the infringement of the patient's rights to information may result in lost chances of informed concent possibly leading to physical or mental damages. In both cases, in essence, the patients lost their chance. Lawsuits of this kind may legally belong to both tort liability and liability for breach of contract, while it tends to be treated as tort liability in practice.

\section{Infringements of rights to know and the loss of chance}

The "loss of chance" as a ground of judgment has been applied in the judicial circles in many countries [5]. So far "loss of chance" remains highly controversial both in theory and in forensic practice. Forensic medical experts seek to identify causation based on case facts and epidemiological knowledge. The use of the theory "loss of chance" is highly recommended to analyze and identify causation. According to the theory, when it comes to the loss of chance, what we lose is an opportunity or a possibility. When forensic experts evaluate medical damages caused by infringement of patient information as well as the loss of chance for compensation, it is necessary to pay attention to the following points:

(1) The patient's right to be informed should be practiced under the current medical treatments, health law, administrative rules, departmental regulations, diagnosis and treatment standards and ethical principles.

(2) The right should also include information about the development of the disease and the treatment possibilities. The truth shouldn't be concealed in the name of confidentiality or privacy.

(3) The opportunity lost is an objective which should be affirmed under the current medical treatments, health law, administrative rules, departmental regulations, diagnosis and treatment standards and large sample epidemiological investigations.

(4) The grounds for appeal that a treatment is still at the experimental stage or in clinical trial should not be supported.

(5) If damage did not occur then the patient should not appeal for theoretical damages.

(6) Unless the number of causes for the medical damage is definite, the forensic expert shouldn't exclude other possible reasons however unlikely.

Since chief parties in both cases infringed on the patients' rights to be informed and caused their loss of chance, the identification of the causation should follow the principles above. For example, we should take the current medical literature into consideration so that we can identify the extent of the loss of chance and refer evidencebased judgments on causation and degree of contribution.

\section{Identification principles of corresponding degree}

According to data from the World Health Organization, International Classification of Disability and Health [6], the degree of contribution of medical damage caused by medical fault fall into the following types:

As mentioned above, the infringements of the patients' rights to be informed reduces or annihilate the patients' chance of precautionary measures and further treatment. It is necessary to perform large sample epidemiological investigations related to loss of chance in order to analyze whether a timely information would have had a positive impact on the patient's condition.

In the first case, medical faults made by the surgeon of the provincial hospital are as follows:

(1) The diagnosis of the suspected gallbladder lesion had been made prior to the operation. However, the surgeon misdiagnosed it and performed a cholecystectomy.

(2) The surgeon did not pay attention to the pathological result. It was according to the report from the intraoperative frozen section biopsy he ruled out the possibility of carcinoma of gallbladder.

(3) The most critical factor was that the surgeon failed to inform the patient of the pathological result in time.

This resulted in the patient's loss of chance to have radical surgery. As mentioned above, the carcinoma of the gallbladder was at an early stage and the overall 5 year survival rate was reported as $12 \%-34 \%$. 
Citation: Shu Z, Jie-Min C, Dong-Mei L, Shu-Ya P, Wen-Tao X. Identification of Causation between Misdiagnosis and Death by the "Loss of Chance" Theory: Two Case Reports. J Forensic Investigation. 2015;3(1): 5.

Epidemiologically, even if a radical operation was performed, total recovery would be unlikely. On the basis of the principles of degree of contribution mentioned above, the misdiagnosis is identified as a contributory cause and the degree of contribution for the provincial hospital should be between $20 \%$ and $40 \%$.

In the second case, the neglect of registering pancreatic abnormalities on the CT scan made the patient loose his chance of further examination and a definite diagnosis. However, pancreatic carcinoma is highly malignant. Even if the definite diagnosis had been properly made and the radical surgery had been successfully performed, the recovery rate as well as the survival rate would remain extremely low. So, the forensic medical expert eventually concluded that the medical fault was a predisposing factor of death and the degree of contribution should be between $10 \%$ and $15 \%$.

In brief, many relative factors should be considered in such cases. These factors include individual factors like co-morbidity, sex and age as well as factors such as limitations of medical knowledge and medical fault such as diagnosis or treatment. Forensic medical experts should include all of these factors in their analysis to conduct an evidence-based assessment of "loss of chance".

\section{References}

1. Friedman SL, McQuaid KR, Grendell JH (2003) Current diagnosis \& treatment in gastroenterology, United States of America: Longman, p. 315-319.

2. Principle A, DelGaudio M, Ercolani G, Golfieri R, Cucchetti A, et al. (2006) Radical surgery for gallbladder carcinoma: possibilities of survival. Hepatogastroenterology 53: 660-664.

3. Tsitouridis I, Diamantopoulou A, Michaelides M, Arvanity M, Papaioannou S (2010) Pancreatic metastases: CT and MRI findings. Diagn Interv Radiol 16: 45-51.

4. Cleary SP, Gryfe R, Guindi M, Greig P, Smith L, et al. (2004) Prognostic factors in resected pancreatic adenocarcinoma: analysis of actual 5-year survivors. J Am Coll Surg 198: 722-731.

5. King JH (1981) Causation, valuation, and chance in personal injury torts involving preexisting conditions and future consequences. Yale Law J 90: 1353-1397.

6. WHO (2014) International Classification of Functioning, Disability and Health (ICF). 